\title{
Oromucosal Drops Dosage Form
}

National Cancer Institute

\section{Source}

National Cancer Institute. Oromucosal Drops Dosage Form. NCI Thesaurus. Code C149734.

Liquid, usually multidose preparation consisting of a solution, suspension or emulsion intended for oromucosal use. Oromucosal drops are administered by instillation into the oral cavity or onto a specific part of the oral cavity. 